Self-sustained spin-polarized current oscillations in multiquantum well structures

This article has been downloaded from IOPscience. Please scroll down to see the full text article.

2009 New J. Phys. 11013033

(http://iopscience.iop.org/1367-2630/11/1/013033)

View the table of contents for this issue, or go to the journal homepage for more

Download details:

IP Address: 193.144.185.28

The article was downloaded on 05/02/2013 at 17:45

Please note that terms and conditions apply. 


\title{
Self-sustained spin-polarized current oscillations in multiquantum well structures
}

\author{
Ramón Escobedo ${ }^{1,5}$, Manuel Carretero ${ }^{2,3}$, Luis L Bonilla ${ }^{2,3}$ \\ and Gloria Platero 4 \\ ${ }^{1}$ Departamento de Matemática Aplicada y Ciencias de la Computación, \\ Universidad de Cantabria, 39005 Santander, Spain \\ ${ }^{2}$ G. Millán Institute, Fluid Dynamics, Nanoscience and Industrial Mathematics, \\ Universidad Carlos III de Madrid, 28911 Leganés, Spain \\ ${ }^{3}$ Unidad Asociada al Instituto de Ciencia de Materiales de Madrid, CSIC \\ ${ }^{4}$ Instituto de Ciencia de Materiales, CSIC, 28049 Cantoblanco, Spain \\ E-mail: escobedo@unican.es, manuel.carretero@uc3m.es, \\ bonilla@ing.uc3m.es and gplatero@icmm.csic.es \\ New Journal of Physics 11 (2009) 013033 (19pp) \\ Received 20 May 2008 \\ Published 20 January 2009 \\ Online at http://www.njp.org/ \\ doi:10.1088/1367-2630/11/1/013033
}

\begin{abstract}
Nonlinear transport through diluted magnetic semiconductor nanostructures is investigated. We have considered a II-VI multiquantum well nanostructure whose wells are selectively doped with $\mathrm{Mn}$. The response to a dc voltage bias may be either a stationary or an oscillatory current. We have studied the transition from stationary to time-dependent current as a function of the doping density and the number of quantum wells. Analysis and numerical solution of a nonlinear spin transport model shows that the current in a structure without magnetic impurities is stationary, whereas current oscillations may appear if at least one well contains magnetic impurities. For long structures having two wells with magnetic impurities, a detailed analysis of nucleation of charge dipole domains shows that self-sustained current oscillations are caused by repeated triggering of dipole domains at the magnetic wells and motion towards the collector. Depending on the location of the magnetic wells and the voltage, dipole domains may be triggered at both wells or at only one. In the latter case, the well closer to the collector may inhibit domain motion between the first and the second well inside the structure. Our study could allow design of oscillatory spin-polarized current injectors.
\end{abstract}

\footnotetext{
${ }^{5}$ Author to whom any correspondence should be addressed.
} 


\section{Contents}

1. Introduction 2

2. Theoretical model 3

3. Stationary states and self-sustained oscillations 5

3.1. Phase diagram . . . . . . . . . . . . . . . . . . 5

3.2. Current-voltage characteristics . . . . . . . . . . . . . 8

4. Self-oscillations $\quad 10$

4.1. Nucleation of dipole waves at a magnetic well . . . . . . . . . . . . . . . 10

4.2. Multiquantum well structure with two magnetic quantum wells . . . . . . . . 15

4.3. Short multiquantum well structure . . . . . . . . . . . . . 16

5. Conclusions 17

$\begin{array}{lr}\text { Acknowledgments } & 18\end{array}$

$\begin{array}{lr}\text { References } & 18\end{array}$

\section{Introduction}

Impressive success of spintronic applications has been typically realized in metal-based structures which utilize magnetoresistive effects for substantial improvements in the performance of computer hard drives and magnetic random access memories [1]. Correspondingly, the theoretical understanding of spin-polarized transport is usually restricted to the metallic regime in linear response, which, while providing a good description for data storage and magnetic memory devices, is not sufficient for signal processing and digital logic. In contrast, much less is known about possible applications of semiconductor-based spintronics and spin-polarized transport in related structures which could utilize strong intrinsic nonlinearities in current-voltage characteristics to implement spin-based logic. Semiconducting materials offer the possibility of new device functionalities not realizable in metalic systems. Equilibrium carrier densities can be varied through a wide range of doping. In heterostructures and quantum dots, nanosecond spin dynamics persist at room temperature. Furthermore, because the typical carrier densities in semiconductors are low compared to metals, electronic properties are easily tunable by gate potentials $[2,3]$.

While optical excitation is used to create spin polarization in most experiments on spin dynamics, electrical injection and detection of spin currents are more appropriate for practical applications. The large mismatch in conductivity and spin relaxation time between metals and semiconductors produces very small efficiencies in ferromagnetic/semiconductor junctions when used as spin injectors [4]. This has spurred the research on diluted magnetic semiconductors (DMS) that can be associated more easily with nonmagnetic semiconductors for spin injection [5, 6]. Using contacts based in $\mathrm{Mn}$, it has been shown that DMS are efficient spin injectors [7]-[10]. Electrical injection of spin-polarized current makes DMS nanostructures interesting potential spintronic devices $[9,11]$. In DMS, spin plays an important role in electron dynamics, particularly in II-VI-based semiconductor superlattices (SL) doped with $\mathrm{Mn}^{2+}$ ions [12]. Carrier-ion exchange spin effects dominate the magneto-transport in these systems, producing spin-polarized transport and large magneto-resistance. Exchange interaction between the spin carrier and $\mathrm{Mn}$ ions results in large spin splittings. In fact, full spin polarization has been achieved at magnetic fields of $1 \mathrm{~T}$. Recently [13]-[15], nonlinear transport through DMS SL has 
been investigated. The interplay between the nonlinearity of the current-voltage characteristics and the exchange interaction produces interesting spin-dependent features: multistability of steady states with different polarization in the magnetic wells [13], time-periodic oscillations of the spin-polarized current and induced spin polarization in nonmagnetic wells by their magnetic neighbors $[14,15]$, among others. The high sensitivity of these systems to external fields points to their potential application as magnetic sensors [13].

In all the previous applications, it is very important to understand in detail nonlinear charge and spin dynamics, which necessarily requires analyzing model equations. In this paper, we analyze nonlinear electron spin dynamics in an n-doped dc voltage biased semiconductor multiquantum well structure (MQWS) having one or more of its wells doped with magnetic impurities (Mn). Provided one quantum well (QW) has been doped with Mn (for example, the QW adjacent to the emitter), we have shown in previous works [15] that spin-polarized current can be obtained using normal contacts. Under dc voltage bias, we have also shown that a sufficiently long MQWS (four or more QWs) may exhibit static electric field domains (EFD) and stationary current or moving domains and self-sustained current oscillations (SSCOs). The existence of SSCOs depends on the doping density, the spin splitting induced by the exchange interaction and the number of QWs [15].

In the present work, we extend our analysis to include a phase diagram of the electric field versus the number of wells for the case of one QW doped with magnetic impurities. We find that there are several voltage intervals for which SSCOs occur although the first interval with lower voltages is the widest. The voltage at the onset of the $k$ th SSCO interval is proportional to $k$ and does not depend on the number of QWs in the structure. Augmenting spin splitting by tuning the external magnetic field, we find that higher SSCO intervals are absorbed by the first one, whose width correspondingly increases. We also characterize the bias regions where SSCOs occur. We analyze in detail the nucleation of dipole waves at a magnetic well and also wave generation and propagation when more than one well is doped with magnetic impurities. We show how at certain voltages simultaneous periodic nucleation from two magnetic wells occurs. Finally, a detailed analysis shows the minimal doping density for SSCOs for different numbers of QWs. We also characterize how the nonlinear electron dynamics is affected by the following factors: number of wells, density doping, splitting of energy subbands due to the magnetic field, number of magnetic wells and their distribution within the sample. These factors are very important for designing a spin-polarized current oscillator.

\section{Theoretical model}

The sample under consideration consists of an n-doped $\mathrm{ZnSe} /(\mathrm{Zn}, \mathrm{Cd}, \mathrm{Mn}) \mathrm{Se}$ weakly coupled MQWS. The spin for the magnetic ion $\mathrm{Mn}^{2+}$ is $S=\frac{5}{2}$ and the exchange interaction between the Mn local moments and the conduction band electrons is ferromagnetic in II-VI QWs. Using the virtual crystal and mean field approximations, the effect of the exchange interaction is to make the sub-band energies spin dependent in those QWs that contain Mn ions: $E_{j}^{ \pm}=$ $E_{j} \mp \Delta / 2$ where $\Delta=2 J_{\mathrm{sd}} N_{\mathrm{Mn}} S B_{\mathrm{S}}\left(g \mu_{\mathrm{B}} B S /\left(k_{\mathrm{B}} T_{\text {eff }}\right)\right)$ for electronic spin $s= \pm \frac{1}{2}$, and $B, J_{\mathrm{sd}}$, $N_{\mathrm{Mn}}, k_{\mathrm{B}}$ and $T_{\text {eff }}$ are the external magnetic field, the exchange integral, the density of magnetic impurities, the Boltzmann constant and an effective temperature which accounts for $\mathrm{Mn}$ interactions, respectively $[13,16]$. We model spin-flip scattering coming from spin-orbit or hyperfine interaction by means of a phenomenological scattering time $\tau_{\text {sf }}$, which is larger than impurity and phonon scattering times: $\tau_{\text {scat }}=\hbar / \gamma<\tau_{\text {sf }}$. Vertical transport in the nanostructure is 
spin-independent sequential tunneling between adjacent QWs, so that when electrons tunnel to an excited state they instantaneously relax by phonon scattering to the ground state with the same spin polarization. Lastly, electron-electron interaction is considered within the Hartree mean field approximation.

The equations describing our model are [15]

$$
\begin{aligned}
& F_{i}-F_{i-1}=\frac{e}{\varepsilon}\left(n_{i}^{+}+n_{i}^{-}-N_{\mathrm{D}}\right), \\
& e \frac{\mathrm{d} n_{i}^{ \pm}}{\mathrm{d} t}=J_{i-1 \rightarrow i}^{ \pm}-J_{i \rightarrow i+1}^{ \pm} \pm \frac{1}{\tau_{\mathrm{sf}}}\left(n_{i}^{-}-\frac{n_{i}^{+}}{1+\mathrm{e}^{\left(E_{1, i}^{-}-\mu_{i}^{+}\right) / \gamma_{\mu}}}\right),
\end{aligned}
$$

where $i=1, \ldots, N . n_{i}^{+}, n_{i}^{-}$and $-F_{i}$ are the two-dimensional (2D) spin-up and spin-down electron densities, and the average electric field at the $i$ th SL period (which starts at the right end of the $(i-1)$ th barrier and finishes at the right end of the $i$ th barrier), respectively.

The voltage bias condition for the applied voltage $V$ is

$$
\sum_{i=0}^{N} F_{i} l=V .
$$

We have denoted the spin-dependent subband energies $(\mathcal{E})$ (measured from the bottom of the $i$ th well) by $E_{j, i}^{ \pm}=E_{j} \mp \Delta_{i} / 2$, with $\Delta_{i}=\Delta$ or 0 , depending on whether the $i$ th well contains magnetic impurities. $N_{\mathrm{D}}, \varepsilon,-e, l=d+w$, and $-J_{i \rightarrow i+1}^{ \pm}$are the $2 \mathrm{D}$ doping density at the QWs, the average permittivity, the electron charge, the width of a SL period ( $d$ and $w$ are barrier and well widths) and the tunneling current density across the $i$ th barrier, respectively.

For electrons with spin $\pm \frac{1}{2}$, the chemical potentials at the $i$ th SL period, $\mu_{i}^{ \pm}$, are related to the electron densities by

$$
n_{i}^{ \pm}=\rho \ln \left[1+\exp \left(\frac{\mu_{i}^{ \pm}-E_{1, i}^{ \pm}}{k_{\mathrm{B}} T}\right)\right],
$$

where $\rho=m^{*} k_{\mathrm{B}} T /\left(2 \pi \hbar^{2}\right)$ and $m^{*}$ is the effective electron mass.

For numerical convenience, the right-hand side of (2) contains a smoothed form of the scattering term used in [13]. As $\gamma_{\mu} \rightarrow 0$, our scattering term becomes $\pm\left(n_{i}^{-}-n_{i}^{+}\right) / \tau_{\mathrm{sf}}$ for $\mu_{i}^{+}>E_{1, i}^{-}$(equivalently, $\mu_{i}^{+}-E_{1, i}^{+}>\Delta$ ), and $\pm n_{i}^{-} / \tau_{\text {sf }}$ otherwise [13].

Time-differencing equation (1) and inserting (2) into the result, the following form of Ampère's law is obtained

$$
\varepsilon \frac{\mathrm{d} F_{i}}{\mathrm{~d} t}+J_{i \rightarrow i+1}=J(t)
$$

where $J_{i \rightarrow i+1}=J_{i \rightarrow i+1}^{+}+J_{i \rightarrow i+1}^{-}$and $J(t)$ is the total current density. The total current density $J(t)$ is independent of $i$, as it can be written as

$$
J(t)=\frac{1}{N+1} \sum_{i=0}^{N} J_{i \rightarrow i+1}
$$

by adding (5) for all $i$ and using that time-differencing equation (3) yields

$$
\frac{\mathrm{d} V}{\mathrm{~d} t}=l \sum_{i=0}^{N} \mathrm{~d} F_{i} / \mathrm{d} t=0 .
$$


Tunneling currents $J_{i \rightarrow i+1}^{ \pm}$are calculated by the transfer Hamiltonian method taking into account that spin-up and -down electrons have different energies:

$$
J_{i \rightarrow i+1}^{ \pm}=\frac{e v^{(f) \pm}\left(F_{i}\right)}{l}\left\{n_{i}^{ \pm}-\rho \ln \left[1+\mathrm{e}^{\left(-e F_{i} l\right) /\left(k_{\mathrm{B}} T\right)}\left(\mathrm{e}^{n_{i+1}^{ \pm} / \rho}-1\right)\right]\right\}
$$

for $i=1, \ldots, N-1$, provided that scattering-induced broadening of energy levels is much smaller than sub-band energies and chemical potentials [17, 18].

The spin-dependent 'forward tunneling velocity' $v^{(f) \pm}$ is a sum of Lorentzians of width $2 \gamma$ (the same value for all sub-bands, for simplicity) centered at the resonant field values $F_{j, i}^{ \pm}=\left(E_{j, i+1}^{ \pm}-E_{1, i}^{ \pm}\right) /(e l)[17]$ :

$$
v^{(f) \pm}\left(F_{i}\right)=\frac{\hbar^{3} l \gamma}{2 \pi^{2} m^{* 2}} \sum_{j=1}^{2} \frac{\mathcal{T}_{i}\left(E_{1, i}^{ \pm}\right)}{\left(E_{1, i}^{ \pm}-E_{j, i+1}^{ \pm}+e F_{i} l\right)^{2}+(2 \gamma)^{2}} .
$$

Here, $\mathcal{T}_{i}$ is proportional to the dimensionless transmission probability across the $i$ th barrier [17].

The tunneling current density $J_{i \rightarrow i+1}=J_{i \rightarrow i+1}^{+}+J_{i \rightarrow i+1}^{-}$is a function of $F_{i}, n_{i}^{ \pm}$and $n_{i+1}^{ \pm}$. For constant values $n_{i}^{ \pm}=N_{\mathrm{D}} / 2$ and $F_{i}=F$, the tunneling current density at a nonmagnetic QW has a maximum $J_{\mathrm{M}}$ at a value $F_{\mathrm{M}}$ of the field. In terms of $F_{\mathrm{M}}$, the voltage bias condition can be written as a condition for the average field $\phi$ :

$$
\phi \equiv \frac{V}{(N+1) F_{\mathrm{M}} l}=\frac{1}{(N+1) F_{\mathrm{M}}} \sum_{i=0}^{N} F_{i} .
$$

As boundary tunneling currents for $i=0$ and $N$, we use (8) with $n_{0}^{ \pm}=n_{N+1}^{ \pm}=\kappa N_{\mathrm{D}} / 2$ (identical normal contacts with $\kappa \geqslant 1$ ) [13]. Initially, we set $F_{i}=V /[l(N+1)]$ (then $\phi=$ $F_{i} / F_{\mathrm{M}}$ ), and $n_{i}^{ \pm}=N_{\mathrm{D}} / 2$ (normal QWs).

\section{Stationary states and self-sustained oscillations}

For typical values of the parameters, the solutions of the model equations (1)-(9) include a variety of stationary states with EFDs [13] and SSCOs [15]. We have considered a sample with $d=10 \mathrm{~nm}, w=5 \mathrm{~nm}, \tau_{\mathrm{sf}}=10^{-9} \mathrm{~s}$ (normal QWs) [19] and $10^{-11} \mathrm{~s}$ (magnetic QWs) [20], $m^{*}=$ $0.16 m_{0}, \varepsilon=7.1 \varepsilon_{0}, T=5 \mathrm{~K}, E_{1}=15.76 \mathrm{meV}, E_{2}=61.99 \mathrm{meV}, \gamma=1 \mathrm{meV}, \gamma_{\mu}=0.1 \mathrm{meV}$ and $\kappa=1$. Only the first QW contains magnetic impurities yielding a spin splitting $\Delta=12 \mathrm{meV}$. This value is lower than $\Delta=15 \mathrm{meV}$ used in [15]. In that reference, the first voltage interval for which SSCOs are stable is quite wide and no other voltage intervals of SSCOs were described. Using $\Delta=12 \mathrm{meV}$ provides narrower and more numerous regions of SSCOs, and this significantly affects the MQWS dynamics. For voltages in the first oscillatory interval, the features of SSCOs are quite similar for $\Delta=12$ or $15 \mathrm{meV}$ and whatever is said of them for one value of $\Delta$ holds for the other.

\subsection{Phase diagram}

As explained in [15], SSCOs appear when the doping density at the QWs surpasses a first critical value which depends on the number of wells $N$ as $N_{\mathrm{D}, 1}=2 /(N-2) \times 10^{10} \mathrm{~cm}^{-2}$. For 


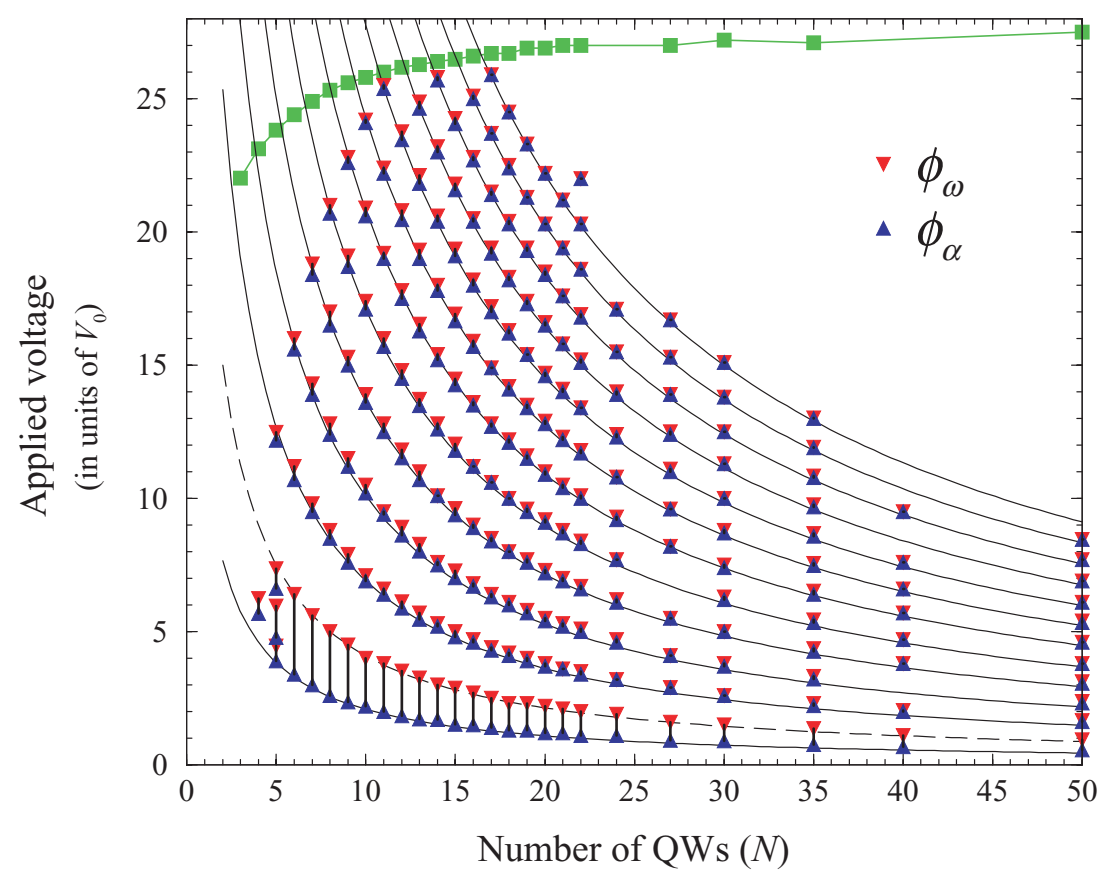

Figure 1. Phase diagram of average electric field $\phi=V /\left[F_{\mathrm{M}}(N+1) l\right]$ versus $N$ for a MQWS containing magnetic impurities only in its first QW and having $N_{\mathrm{D}}=10^{10} \mathrm{~cm}^{-2}, F_{\mathrm{M}}=0.64 \mathrm{kV} \mathrm{cm}-1, J_{\mathrm{M}}=0.409 \mathrm{~A} \mathrm{~cm}^{-2}$ and $\Delta=12 \mathrm{meV}$. The SSCOs begin at $\phi=\phi_{\alpha, k}^{N}$ (lines marked with triangles) and end at $\phi=\phi_{\omega, k}^{N}$ (lines marked with inverted triangles). Solid lines are given by formula (11). In the regions of the phase diagram where there are no SSCOs, the stable solutions are stationary states. The upper line joining solid squares marks the bias values at which the current in the current-voltage characteristics drops to a value corresponding to an almost uniform state with zero charge density; see figure 3.

$N_{\mathrm{D}}=10^{10} \mathrm{~cm}^{-2}$, SSCOs appear in several intervals of the average field (10), $\phi_{\alpha, k}^{N}<\phi<\phi_{\omega, k}^{N}$, $k \in[1,2, \ldots]$. The number and width of these intervals of oscillatory solutions depend on $N$, as shown in figure 1, where $\phi_{\alpha, k}^{N}$ and $\phi_{\omega, k}^{N}$ are marked with triangles and inverted triangles, respectively.

We observe that the sequence of $\phi$ at which oscillations appear can be approximated by the formula:

$$
\phi_{\alpha, k}^{N}=\frac{C_{k} k}{N+1}, \quad C_{1}=23, \quad C_{k}=38(k \geqslant 2),
$$

which provides the solid lines in figure 1. Since $\phi$ is an average field, the voltages corresponding to $(11)$ are $(N+1) \phi_{\alpha, k}^{N}$, proportional to $k$ and independent of the number of QWs in the structure. This can be explained by noticing that the structure of stationary states between oscillation regions is as follows. In the voltage interval between the $(k-1)$ th and $k$ th oscillation regions (for $k \geqslant 3$ ), the stationary state has a field profile comprising two EFDs, a low field region close to the injecting contact with almost zero field and a high field region close to the receiving contact where $F_{i} \approx \phi F_{\mathrm{M}}(N+1) / k$, for the last $k$ QWs. Figure 2 depicts the electric field profile for $k=7$, the same value of the voltage and different $N$. We have shifted the profiles so 


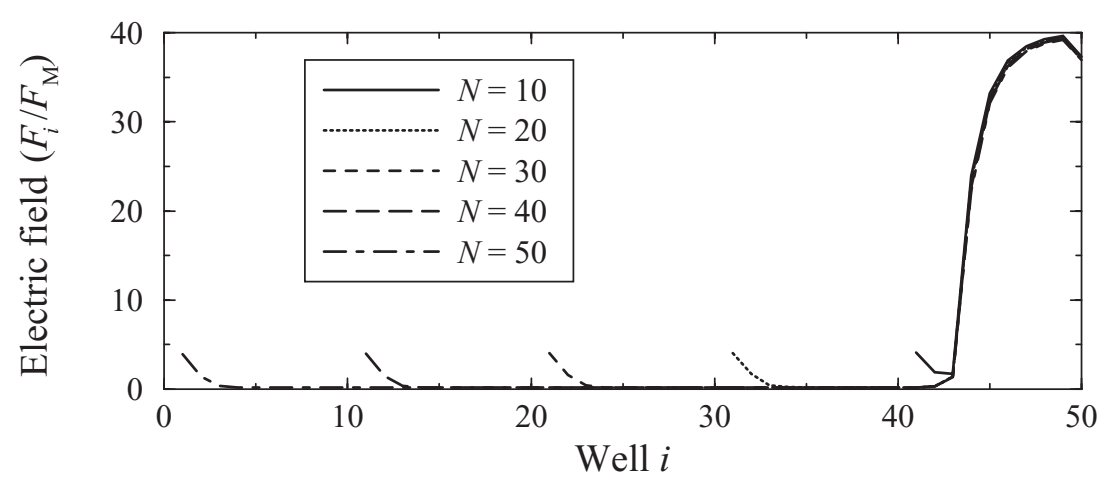

Figure 2. Electric field profile for $\phi_{\omega, 6}^{N}<\phi<\phi_{\alpha, 7}^{N}$ in the seventh interval of stationary solutions, corresponding to $(N+1) \phi=260(38 \times 6<260<38 \times 7)$ and $N=10,20,30,40,50$. Other parameter values are as in figure 1 . We have shifted the profiles so that all the high EFDs end at $i=50$. Note that the high field regions almost coincide for all the values of $N$.

that all finish at $i=50$, which shows that the high field domains coincide for all $N$, thereby confirming the previous description. The values $\phi_{\alpha, k}^{N}$ mark the beginning of the $k$ th oscillation region. At those values the previously described stationary state becomes linearly unstable. Provided the high field domain region contributes the most to the unstable mode, it is plausible that $\phi F_{\mathrm{M}}(N+1) / k$ is almost constant at the instability line, which yields (11) with $C_{k}=38$ for $k \geqslant 2$, after fitting numerically the proportionality constant. The field profile for voltages below the first SSCO interval is almost uniform with $F_{i} \approx 0, i \geqslant 2$, except for a high field value at the first $\mathrm{QW}$ with $F_{1} \approx(N+1) \phi$. Similarly, we expect $(N+1) \phi_{\alpha, 1}^{N}$ to be approximately constant. Fitting yields a different proportionality constant, $C_{1}=23$. The stationary field profiles for voltages larger than the last SSCO interval are again almost uniform with $F_{i} \approx \phi F_{\mathrm{M}}$ for sufficiently large $N$. This agrees with the trend towards a horizontal asymptote for the last SSCO interval observed in figure 1.

Furthermore, both $\phi_{\alpha, k}^{N}$ and $\phi_{\omega, k}^{N}$ decrease as $N$ increases and the width of the intervals, $\left(\phi_{\omega, k}^{N}-\phi_{\alpha, k}^{N}\right)$, decreases as $k$ increases for fixed $N$. For fixed $k$, the width $\left(\phi_{\omega, k}^{N}-\phi_{\alpha, k}^{N}\right)$ decreases as $N$ increases. Note that all the voltage intervals where SSCOs exist (except for the first one with $k=1$ ) are very narrow. The largest value of $\phi$ for which there are SSCOs is about 25 corresponding to $N=17$. It is interesting that if we join the curve $\phi_{\alpha, 1}^{N}$ to the envelope of the minimal $N$ for which SSCOs start and to the envelope of the maximal $\phi_{\omega, k}^{N}$ for each $N$, we obtain a region reminiscent of the region in the phase plane of average electric field versus sample length for which there are Gunn effect oscillations in axisymmetric samples [21].

What happens for other values of $\Delta$, the spin splitting due to the magnetic field? SSCOs are possible only if the splitting surpasses a certain threshold (about $4.06 \mathrm{meV}$ for $N=10$ ). Between this threshold and $\Delta \approx 11 \mathrm{meV}$, different SSCO intervals open up. From this value of $\Delta$ up to a critical value, $\Delta_{\mathrm{c}} \approx 14.2$, the situation is as described above for $\Delta=12 \mathrm{meV}$, except that the width of all the SSCO intervals increases with $\Delta$ and the proportionality constants $C_{k}$ also depend on $\Delta$. For $\Delta>\Delta_{\mathrm{c}}$, the SSCO intervals begin to be absorbed by the first one, which becomes much wider. For $\Delta=15 \mathrm{meV}$ (the value used in [15]), there are between one and three SSCO intervals, depending on $N$. For $6 \leqslant N \leqslant 8$ and $N=17$ there are only two SSCO intervals, three for $N$ between 9 and 16 and only one for $N=5$ and for $N=18$ and larger. In any case, the 


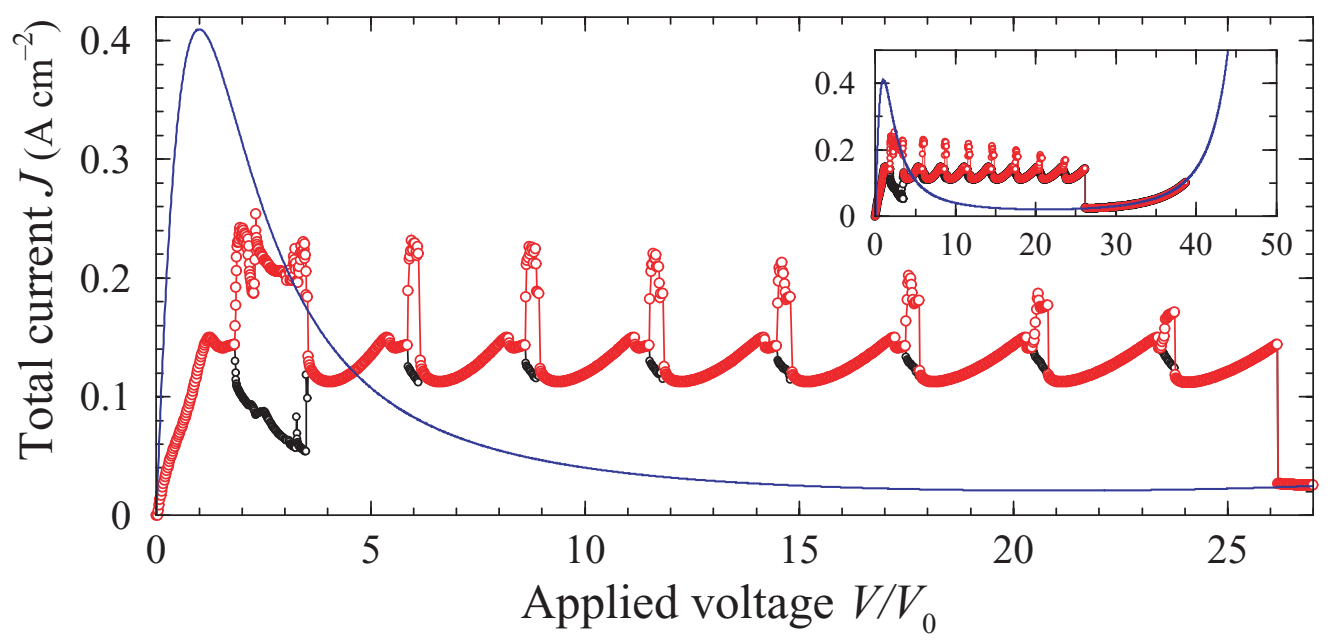

Figure 3. Current-voltage characteristics for the MQWS with $N=12$ and $\Delta=$ $12 \mathrm{meV}$. The maximum and minimum of the SSCOs has been represented in each interval $\left(\phi_{\alpha, k}^{N}, \phi_{\omega, k}^{N}\right)$ with $k=1, \ldots, 8$. The solid line is $J_{i \rightarrow i+1}$ for $F_{i}=V / V_{0}$, $n^{ \pm}=N_{\mathrm{D}} / 2$, with $V_{0}=F_{\mathrm{M}}(N+1) l=12.5 \mathrm{mV}$. Inset: same for a wider voltage range.

voltage at the onset of the first SSCO interval is also approximately constant, $(N+1) \phi_{\alpha, 1}^{N} \approx 33$. If we continue increasing $\Delta$, only the first SSCO interval persists. For example, this occurs at $\Delta=16 \mathrm{meV}$ for $N=10$.

\subsection{Current-voltage characteristics}

Figure 3 depicts the total current density in terms of the dimensionless average field $\phi$ for a fixed doping density $N_{\mathrm{D}}=10^{10} \mathrm{~cm}^{-2}$. In the voltage intervals $\left(\phi_{\alpha, k}^{N}, \phi_{\omega, k}^{N}\right)$ the maximum and minimum values of the current during SSCOs are shown. The stationary states for $\phi<\phi_{\alpha, 1}^{N}$ and for $\phi>\phi_{\omega, 8}^{N}$ (the last oscillation interval) are spatially almost uniform:

$$
\begin{aligned}
\frac{F_{i}}{F_{\mathrm{M}}} & =\phi, \\
J(\phi) & =J_{i \rightarrow i+1}^{+}\left(F_{\mathrm{M}} \phi, q_{1} N_{\mathrm{D}}, q_{1} N_{\mathrm{D}}\right)+J_{i \rightarrow i+1}^{-}\left(F_{\mathrm{M}} \phi, q_{2} N_{\mathrm{D}}, q_{2} N_{\mathrm{D}}\right),
\end{aligned}
$$

where $J_{i \rightarrow i+1}^{ \pm}\left(F_{i}, n_{i}^{ \pm}, n_{i+1}^{ \pm}\right)$is given by $(8)$ and $q_{1}=(1+P) / 2, q_{2}=(1-P) / 2$ are given by $P$, the uniform value of the spin polarization:

$$
P_{i}=\frac{n_{i}^{+}-n_{i}^{-}}{n_{i}^{+}+n_{i}^{-}}
$$

The stationary states for $\phi_{\omega, k}^{N}<\phi<\phi_{\alpha, k+1}^{N}$ (bias between the intervals $k$ and $(k+1)$ of SSCOs) contain two EFDs, a low field domain adjacent to the cathode and a high field domain that extends to the anode, separated by a domain wall in which the field increases. For larger bias, the domain wall moves closer to the injecting contact but domain walls cannot start at the $\mathrm{QW}, i=2$, adjacent to the magnetic $\mathrm{QW}, i=1$, and at $i=N-1$ due to the boundary condition at $i=N$. 
There are $N-2=10$ voltage intervals of stationary solution branches in figure 3, including the almost uniform branches before the first and after the last interval of SSCO. At the low field domain, the spin polarization is $60 \%$, so that the lower energy state is more populated, with $n^{+}=0.8 N_{\mathrm{D}}$, than the higher energy state, with $n^{-}=0.2 N_{\mathrm{D}}$. The spin polarization drops to zero in the QW located after the domain wall and then it increases in the high field domain. In figure 3, we observe that the total current density follows $J_{i \rightarrow i+1}$ with $F_{i}=F$ and $n_{i}^{ \pm}=N_{\mathrm{D}} / 2$ for $\phi>\phi_{\omega, 8}^{12}$ despite the fact that the QW spin polarization at the almost uniform state is $60 \%$, not zero.

At the high fields $F>F_{\mathrm{M}} \phi_{\omega, 8}^{12}$, the second term on the right-hand side of (8) is negligible and we have

$$
J_{i \rightarrow i+1}^{ \pm} \approx \frac{e v^{(f)}\left(F_{i}\right)}{l} n_{i}^{ \pm}
$$

( $i$ and $i+1$ are both normal QWs, therefore $\Delta=0$ in them), so that

$$
J_{i \rightarrow i+1}=J_{i \rightarrow i+1}^{+}+J_{i \rightarrow i+1}^{-} \approx \frac{e v^{(f)}\left(F_{i}\right)}{l}\left(n_{i}^{+}+n_{i}^{-}\right)=\frac{e v^{(f)}\left(F_{i}\right) N_{\mathrm{D}}}{l} .
$$

This is the same result as obtained for $n_{i}^{+}=n_{i}^{-}=N_{\mathrm{D}} / 2$. At low voltages, the second term on the right-hand side of (8) is important and the total current differs from the tunneling current for $n_{i}^{+}=n_{i}^{-}=N_{\mathrm{D}} / 2$, as seen in figure 3 .

The wider voltage interval for which SSCOs exist, $\left[\phi_{\alpha, 1}^{12}, \phi_{\omega, 1}^{12}\right]=[1.83,3.54]$, is shown in figure 4 . There are two subintervals with distinctive features; the time-dependent total current density curves marked with vertical lines in figure 4 are depicted in figure 5.

1. For $\phi \in(1.83,2.30)$, the field profiles corresponding to figures 5 (a) and (b) consist of the repeated nucleation of a charge dipole wave at the magnetic $\mathrm{QW}$, its motion towards the anode and its attenuation and disappearance inside the structure. As the wave loses amplitude, the current increases until a new wave is nucleated. The corresponding field profile is depicted in figure 6(a). This is similar to a Gunn effect in bulk GaAs confined to part of the structure [21]. For $\phi$ close to 1.83 (approximately in the interval $1.83<\phi<$ 1.89), the charge dipole dies down before it is clearly detached from the cathode.

2. For $\phi \in(2.30,3.54)$, the field profiles corresponding to figure 5(c) consist of the repeated nucleation of a charge dipole wave at the magnetic QW, its motion towards the anode, its arrival and growth there, and the appearance of a new wave when the current reaches a critical value. This is similar to the usual Gunn effect in bulk GaAs as figures 6(b) and (c) show [22]. As $\phi$ increases, the travel time of the dipole wave remains about the same, while the time to nucleate a new wave increases. This can be observed by direct comparison of the current $J(t)$ and the field profile in figures 5(c) and 6(b) to those in figures 5(d) and 6(c). Note that the polarization at the end of the MQWS is very low except when a dipole wave arrives there, in which case the polarization increases above $75 \%$; see figure $6(\mathrm{~d})$. The wave nucleation time becomes infinite at $\phi_{\omega, 1}^{N}=3.54$; see figures 5(d) and 6(c). This scenario corresponds to a collision of the branch of SSCO with a homoclinic orbit of the stationary state. The fact that the number of periods in the common time interval of figure 5 decreases as $\phi$ increases illustrates the growth of the oscillation period with $\phi$. 


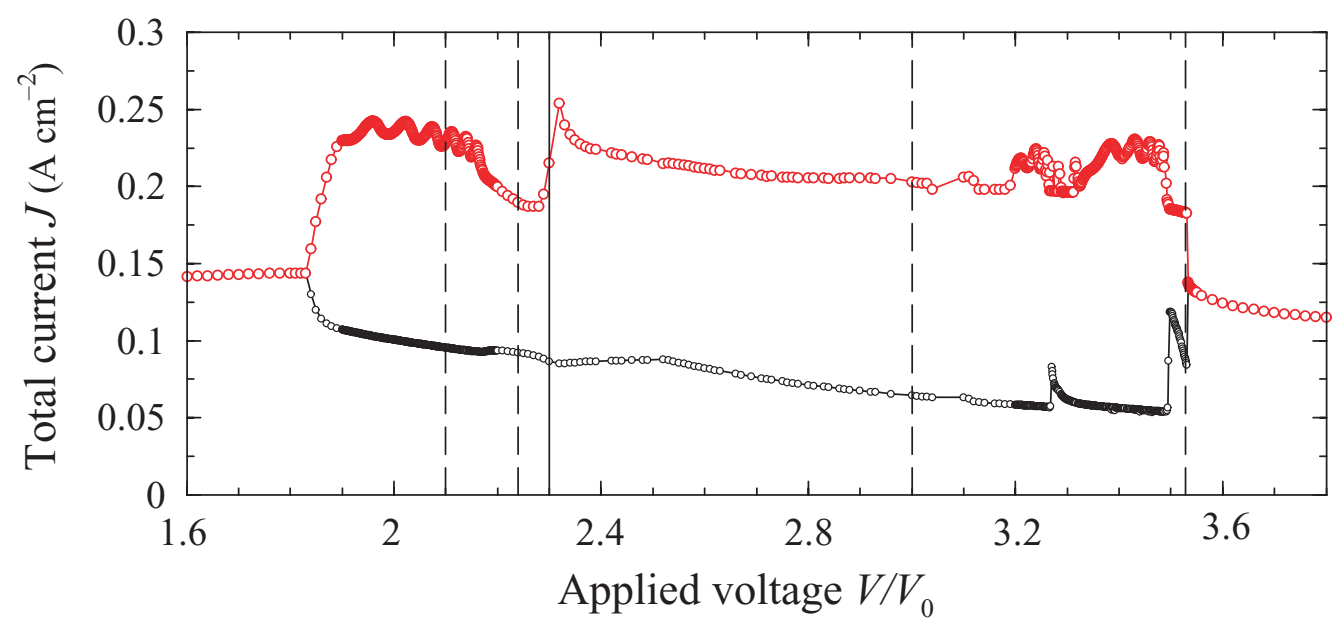

Figure 4. Detail of the current-voltage characteristics for the MQWS with $N=$ 12 corresponding to the first voltage interval of SSCOs in figure $3: \phi_{\alpha, 1}^{12}=1.83$, $\phi_{\omega, 1}^{12}=3.54$. The value $\phi=V / V_{0}=2.3$ marked with a vertical solid line is a critical bias at which a dipole wave triggered at the cathode first arrives at the anode before a second dipole wave is triggered at the cathode. For $\phi<2.3$ dipole waves attenuate and die down before they can arrive at the anode, whereas for $\phi>2.3$ dipole waves arrive and disappear at the anode. The total current density $J(t)$ will be depicted in figure 5 for the bias values marked with vertical dashed lines in this figure.

\section{Self-oscillations}

There are SSCOs for a variety of MQWS configurations, but only if one or more QWs contain magnetic impurities yielding a spin splitting larger than a critical value of a few milli-electronvolt. Nonmagnetic MQWS do not exhibit self-oscillations.

First of all, we have considered long SLs $(N=50)$. For $N$ large, only the first oscillatory region in the current-voltage characteristic curve of figure 3 is sufficiently wide. The corresponding SSCOs are similar to those observed in III-V semiconductor SLs: charge dipoles are triggered at the first well having $\mathrm{Mn}$, move to the collector (near which they may become charge monopoles if $V$ is large enough), disappear there, and new dipoles are triggered [23].

\subsection{Nucleation of dipole waves at a magnetic well}

As shown in figures 7 and 8, if the only magnetic QW is the $I$ th (with $1 \leqslant I<N-3$ ), the charge dipoles are emitted at this well, and dipole motion is limited to the last $N-I$ QWs [15]. Why is this?

Figure 9 depicts the tunneling currents $J_{i \rightarrow i+1}^{ \pm}$and $J_{i \rightarrow i+1}=J_{i \rightarrow i+1}^{+}+J_{i \rightarrow i+1}^{-}$, as functions of $F$ for an uniform field configuration $F_{i}=F$ with $n_{i}^{ \pm}=n_{i+1}^{ \pm}=N_{\mathrm{D}} / 2$. For such a configuration, the solid line is the curve $\mathcal{J}(F)$ corresponding to $J_{i \rightarrow i+1}$ with nonmagnetic $i$ and $i+1$. If $i=I$ is magnetic but $I \pm 1$ are not, the tunneling currents $\mathcal{J}_{I \rightarrow I+1}^{ \pm}, \mathcal{J}_{I \rightarrow I+1}$ and $\mathcal{J}_{I-1 \rightarrow I}$ are also shown in the figure. We will show how these different curves are useful to understand the formation of charge dipole waves at the magnetic field and therefore to understand the origin of SSCOs. 


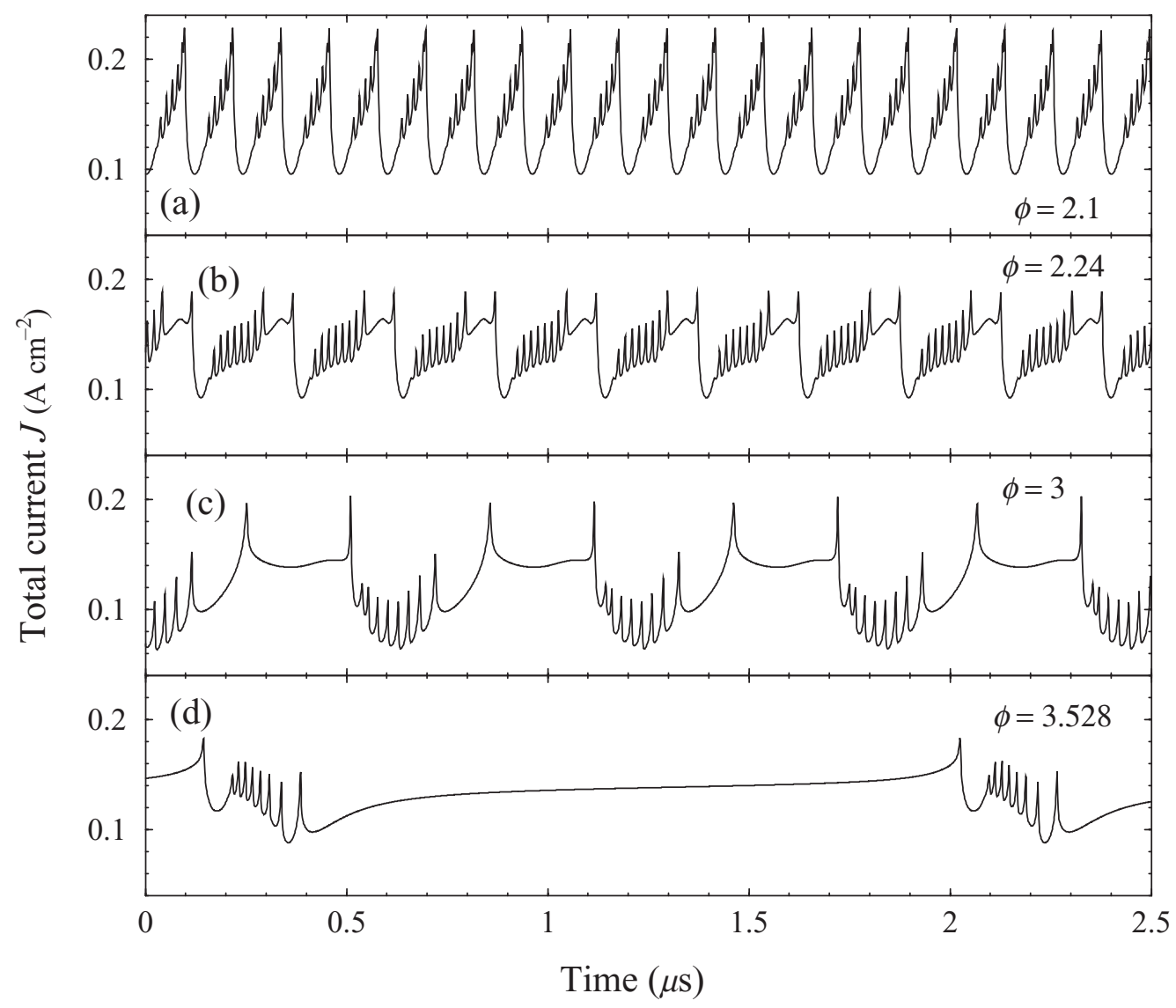

Figure 5. Total current density versus time for the voltages $\phi$ marked with vertical dashed lines in figure 4: (a) 2.1, (b) 2.24, (c) 3.0 and (d) 3.528. Amplitude $\mathcal{A}$ and frequency $v$ of oscillations are as follows: (a) $\mathcal{A}=0.13 \mathrm{~A} \mathrm{~cm}^{-2}$, $v=9.82 \mathrm{MHz}$; (b) $\mathcal{A}=0.095 \mathrm{~A} \mathrm{~cm}^{-2}, v=4.04 \mathrm{MHz}$; (c) $\mathcal{A}=0.15 \mathrm{~A} \mathrm{~cm}^{-2}$, $v=1.76 \mathrm{MHz}$ and (d) $\mathcal{A}=0.095 \mathrm{~A} \mathrm{~cm}^{-2}, v=0.53 \mathrm{MHz}$.

Firstly, we shall explain the shape of the different tunneling current curves in view of their definitions. As $E_{1, i+1}^{ \pm}=E_{1}, E_{1, i}^{ \pm}=E_{1} \mp \Delta / 2$, the first Lorentzian of $v^{(f) \pm}$ in (9) is centered at $F_{1, i}^{ \pm}= \pm \Delta /(2 e l)$. Then $\mathcal{J}_{I \rightarrow I+1}$ has a peak roughly at $\left(\Delta^{2}+8 \gamma^{2}\right) /(2 e l \Delta)$ (if $\left.e F_{\mathrm{M}} l \ll \Delta / 2\right)$, mostly due to $\mathcal{J}_{I \rightarrow I+1}^{+}$. As depicted in figure 9, the height of this peak is under half that of $\mathcal{J}(F)$ (which equals $J_{i \rightarrow i+1}$ for nonmagnetic wells $i$ and $i+1$ ) because $\mathcal{T}_{i}$ is smaller for $E_{1}^{+}$ than for $E_{1}^{-}$. Spin splitting also causes $\mathcal{J}_{I \rightarrow I+1}(F)$ (for magnetic QW $I$ ) to display two peaks at $\left(E_{2}-E_{1} \pm \Delta / 2\right) /(\mathrm{el})$ instead of one peak at $\left(E_{2}-E_{1}\right) /(\mathrm{el})$ with their combined strength in the case of nonmagnetic QWs, $i$ and $i+1$; see figure 1(d) of [15]. If QW $I$ is magnetic, the same argument shows that $\mathcal{J}_{I-1 \rightarrow I}^{ \pm}$has peaks at $\mp \Delta /(2 e l)$ and $\left(E_{2}-E_{1} \mp \Delta / 2\right) /(e l)$, contrary to the shifts in $\mathcal{J}_{I \rightarrow I+1}^{ \pm}$. The shifted curves $\mathcal{J}_{I-1 \rightarrow I}$ and $\mathcal{J}_{I \rightarrow I+1}$ for magnetic $I$ play the role of effective cathode boundary currents during SSCOs. Clearly, they intersect $\mathcal{J}(F)$ (solid line in figure 9) on its second, decreasing branch. One of these intersection points roughly corresponds to the critical current for triggering a new charge dipole, [17, 23, 24]. This is confirmed by the fact that no wave is injected at the cathode if the magnetic QW is well inside the MQWS, as in the case of figure 8 where $I=20$. In this case (with $\kappa=1$ ), the boundary current at 

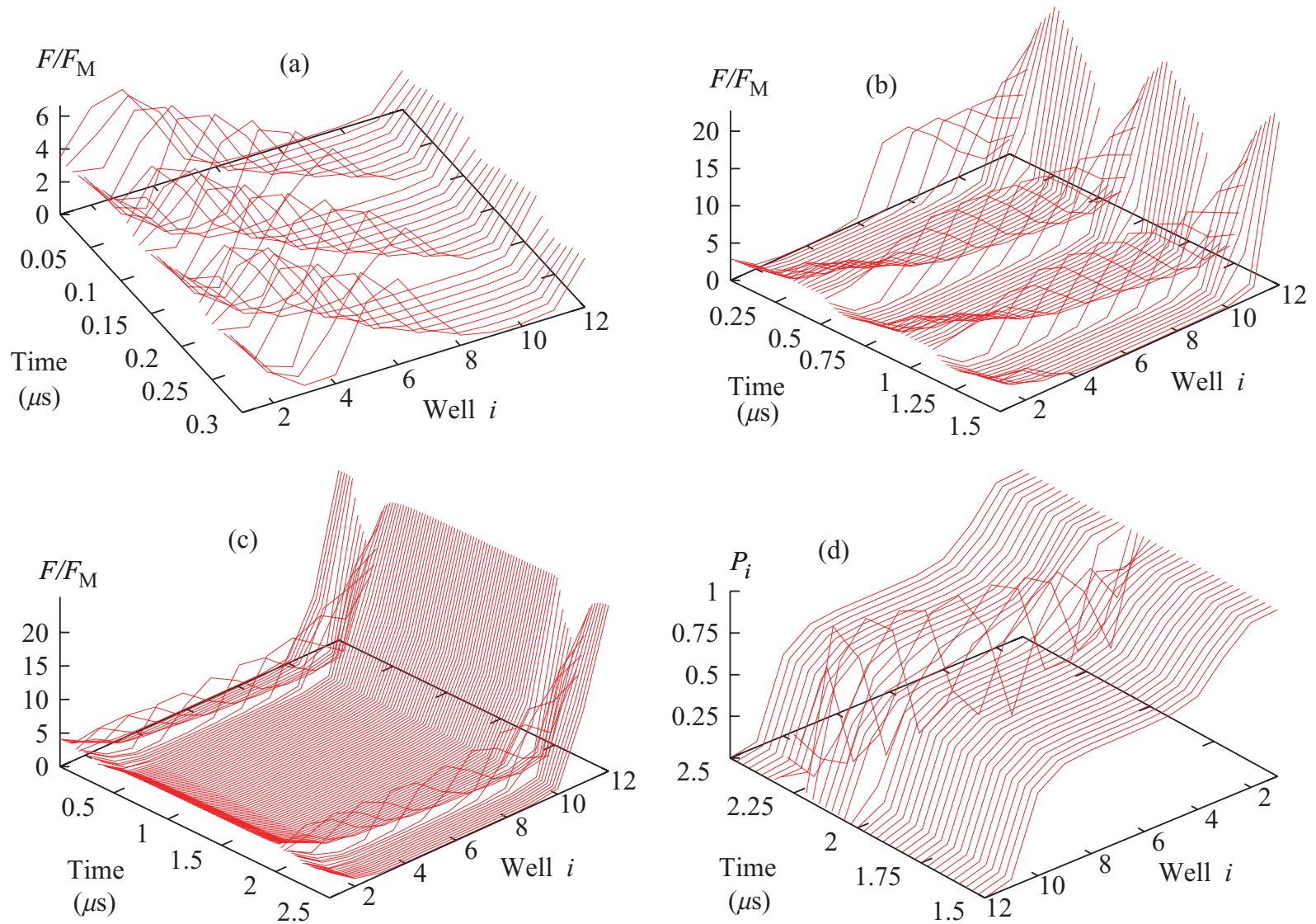

Figure 6. Field profiles for different bias values $\phi=V / V_{0}$ marked in figure 4: (a) 2.1, (b) 3 and (c) 3.528. (d) Polarization profile corresponding to (c). Note the high polarization value at the end of the MQWS as the dipole wave reaches it.

the nonmagnetic injector is $\mathcal{J}(F)$ (the solid line in figure 9), which coincides with the current between nonmagnetic QWs well inside the structure. Such boundary condition precludes current self-oscillations due to dipole recycling. Thus, dipole recycling occurs only for the magnetic and successive QWs, cf figure 8.

Secondly, we want to ascertain which intersection of $\mathcal{J}(F)$ with the other curves in figure 9 corresponds to triggering new dipole waves during SSCOs. In figure 10, we have superimposed to the current tunneling densities a thin solid line displaying $\left(F_{I}(t), J(t)\right)$ and a line with circles displaying $\left(F_{I}, J_{I \rightarrow I+1}\right)$ for all times during one SSCO. These lines intersect $\mathcal{J}(F)$ a number of times. Both graphs, $\left(F_{I}(t), J(t)\right)$ and $\left(F_{I}, J_{I \rightarrow I+1}\right)$, contain a number of loops (bigger and more evident for the first of these graphs than for the second) which correspond to the spikes in the time evolution of $J(t), F_{I}(t)$ and $J_{I \rightarrow I+1}$ depicted in figures 11(a) and (b). In fact, during each oscillation period, $J(t)$ exhibits a series of spikes that correspond to well-to-well hopping of the domain wall located at the back of the moving dipole wave. The spikes with smaller local minima of $J(t)$ correspond to a dipole wave detached from the magnetic field and the anode. When the wave arrives at the anode, the local minima of $J(t)$ take on larger values whereas the maximum field at the exiting wave also increases. The graphs of the functions $F_{I}(t)$ and $J_{I \rightarrow I+1}$ versus time are similar to that of $J(t)$; cf figures $11(\mathrm{a})$ and (b). 

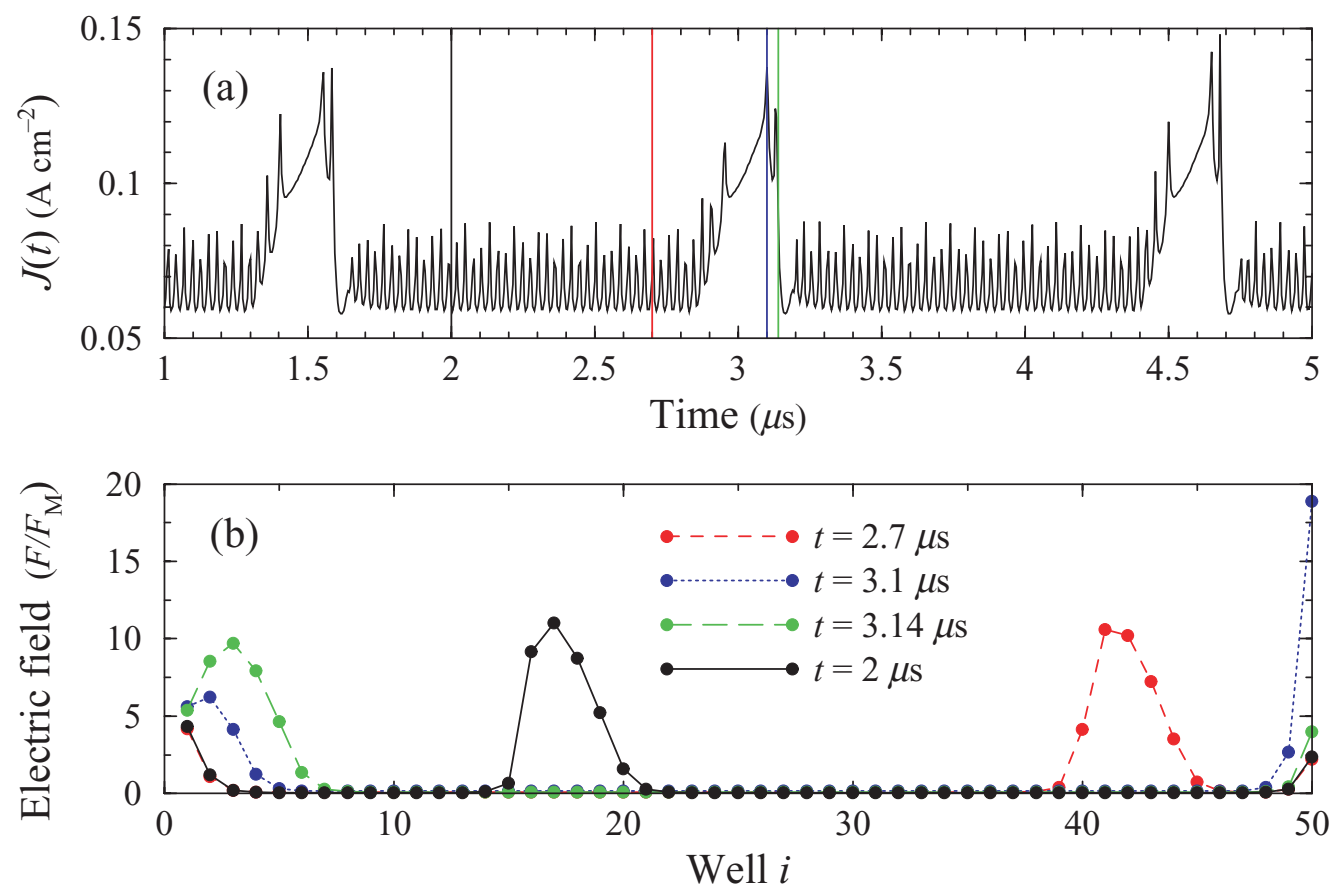

Figure 7. (a) Time periodic total current density $J(t)$ and (b) electric field profiles versus QW index for $N=50, V=0.048 \mathrm{~V}, N_{\mathrm{D}}=10^{10} \mathrm{~cm}^{-2}, F_{\mathrm{M}}=$ $0.64 \mathrm{kV} \mathrm{cm}^{-1}, J_{\mathrm{M}}=0.409 \mathrm{~A} \mathrm{~cm}^{-2}$ and $\Delta=15 \mathrm{meV}$ when the magnetic QW is $i=1$.
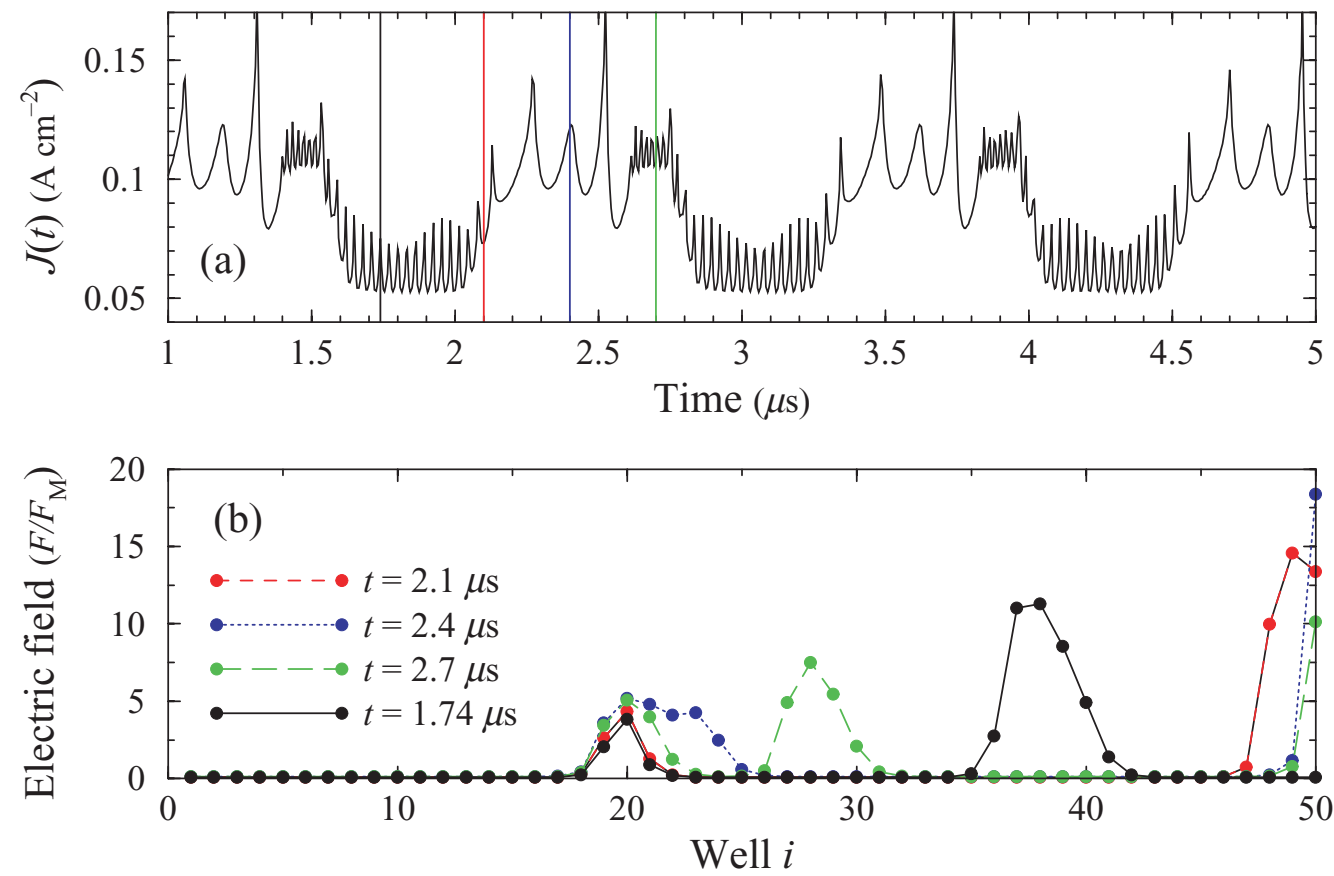

Figure 8. Same as figure 7 when the magnetic QW is $i=20$. 


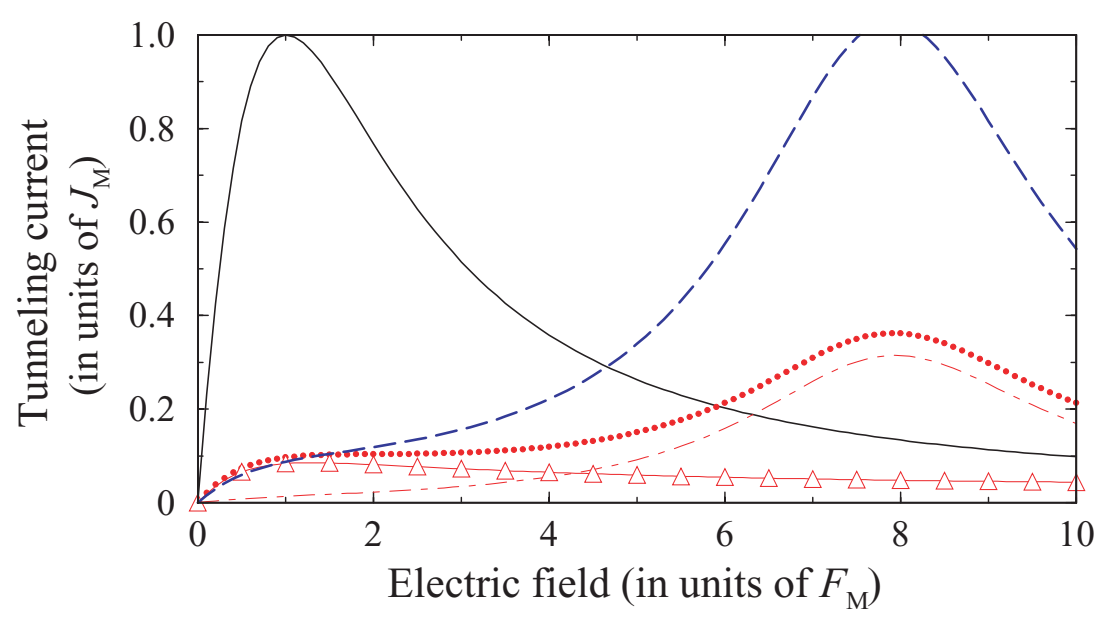

Figure 9. Current densities versus field in an uniform configuration $F_{i}=F$, $n_{i}^{ \pm}=N_{\mathrm{D}} / 2$. Solid line: $\mathcal{J}(F)=J_{i \rightarrow i+1}$ for nonmagnetic $i$ and $i+1$. For magnetic $i=I$, nonmagnetic $I \pm 1: J_{I \rightarrow I+1}$ (dotted line), $J_{I \rightarrow I+1}^{+}$(dot-dashed line), $J_{I \rightarrow I+1}^{-}$ (triangles), $J_{I-1 \rightarrow I}$ (dashed line). In this figure, $N=30, \phi=2, N_{\mathrm{D}}=10^{10} \mathrm{~cm}^{-2}$, and the only QW containing magnetic impurities is $I=5$ with $\Delta=15 \mathrm{meV}$.

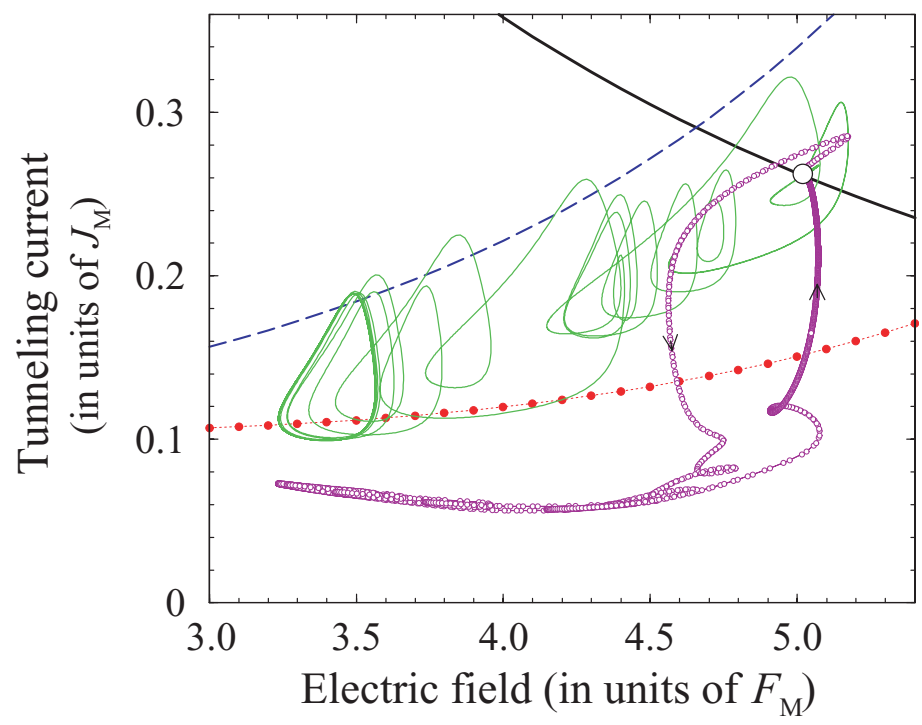

Figure 10. Zoom of the region of figure 9 where the tunneling currents $\mathcal{J}_{I \rightarrow I+1}(F)$ (dotted line) and $\mathcal{J}_{I-1 \rightarrow I}(F)$ (dashed line) intersect $\mathcal{J}(F)$ (solid line). We have superimposed the graphs of $\left(F_{I}(t), J(t)\right)$ (thin solid line) and $\left(F_{I}(t), J_{I \rightarrow I+1}(t)\right)$ (circles) for times during one oscillation period. The arrows in the latter graph indicate the direction of increasing time. In the point marked with an open circle, these two graphs intersect $\mathcal{J}(F)$.

For each time $t$, the tunneling current from the magnetic QW, $J_{I \rightarrow I+1}(t)$, is typically smaller than $J(t)$ except when new dipoles are nucleated. Figure 11(c) shows details of the curves $J(t)$ and $J_{I \rightarrow I+1}$ during the time interval in which they intersect for a magnetic QW located at $I=5$. After a pronounced current spike in figure 11(a) with $J(t)>J_{I \rightarrow I+1}(t)$, there is a region of 

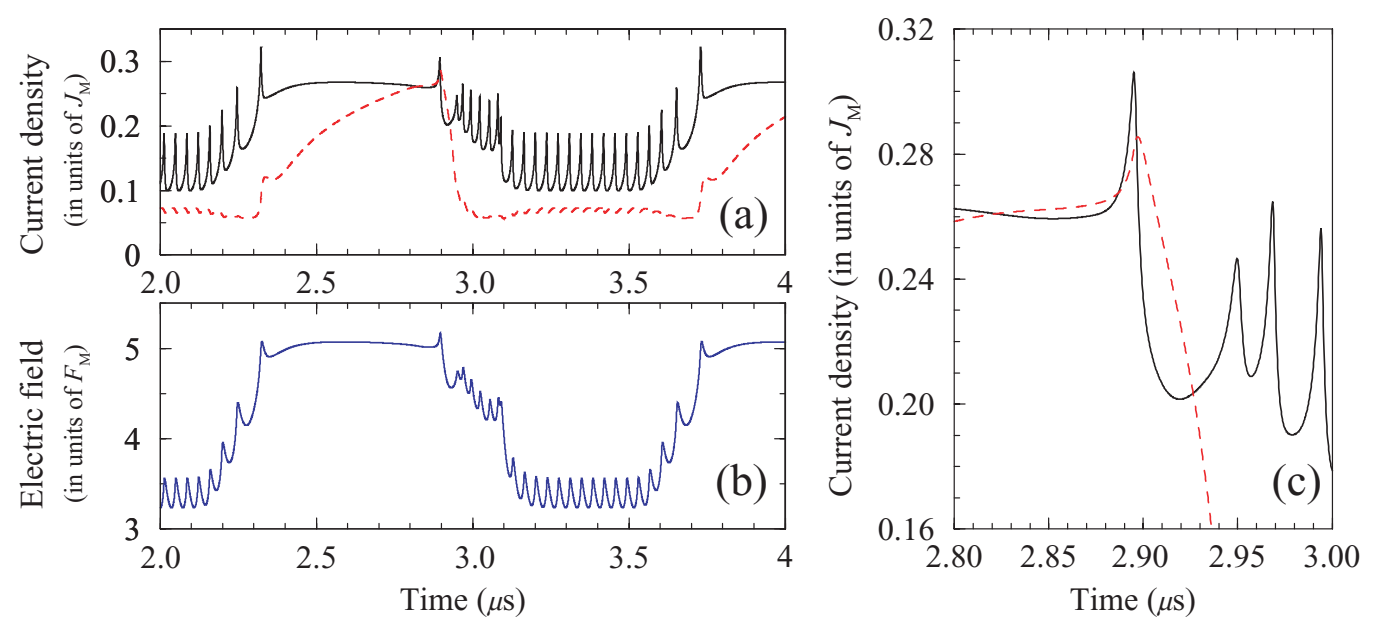

Figure 11. (a) Current densities $J(t)$ (solid line) and $J_{I \rightarrow I+1}(t)$ (dashed line) versus time. (b) Electric field at the magnetic QW, $F_{I}(t)$. (c) Zoom of (a) showing the four intersections between $J(t)$ and $J_{I \rightarrow I+1}(t)$. Parameter values are as in figure 9.

flat current (and rising $J_{I \rightarrow I+1}(t)$ ) that ends at another tall spike of both graphs. Just before this second current spike, $J_{I \rightarrow I+1}(t)$ intersects $J(t)$ twice, as depicted in figure 11(c). After the maximum of the current at this second spike, $J_{i \rightarrow i+1}$ decreases and intersects $J(t)$ twice again, as figure 11(c) shows. At the first two intersection times, the total current density $J(t)$ takes on values that coincide with $\mathcal{J}\left(F_{I}(t)\right)$ on the second branch of $\mathcal{J}(F)$, as shown in figure 10: see the intersection of the thin line $\left(F_{I}, J\right)$, the curve marked with circles, $\left(F_{I}, J_{I \rightarrow I+1}\right)$, and $\mathcal{J}(F)$. The first time that this triple intersection of $\mathcal{J}\left(F_{I}(t)\right), J(t)$ and $J_{I \rightarrow I+1}$ occurs (second intersection of $\left(F_{I}, J_{I \rightarrow I+1}\right)$ and $\mathcal{J}(F)$ at $t_{1}=2.890 \mu \mathrm{s}, J=0.26 J_{\mathrm{M}}$ and $F_{5}=5.02 F_{\mathrm{M}}$, corresponding to the open circle in figure 9) marks the nucleation of a new dipole wave at the magnetic QW. The third time that $J(t)=J_{I \rightarrow I+1}(t)\left(t_{2}=2.930 \mu \mathrm{s}, J=0.2 J_{\mathrm{M}}\right.$ and $\left.F_{5}=4.56 F_{\mathrm{M}}\right)$ marks the release of the new dipole wave from the magnetic QW. After a new wave is created, the local minima of the current decrease while the voltage under the new dipole wave grows at the expense of the shrinking dipole wave that exits at the anode.

Recapitulating, we have observed that nucleation of a new dipole corresponds to the intersection of $J_{I \rightarrow I+1}(t), J(t)$ and $\mathcal{J}\left(F_{I}(t)\right)$, whereas dipole detachment corresponds to the intersection of tunneling and total currents at a current lower than $\mathcal{J}\left(F_{I}(t)\right)$. Figure 9 shows that the total current at the nucleation time, $J\left(t_{1}\right)$, is somewhat lower than that corresponding to the intersection of the graphs of $\mathcal{J}(F)$ and of $\left(F, \mathcal{J}_{I \rightarrow I+1}\right)$ (for magnetic $I$ and normal $I+1$, with equal field $F_{i}=F$ and electron densities $n_{i}^{ \pm}=N_{\mathrm{D}} / 2$ at all QWs). This is not surprising: as the field configuration $F_{i}$ is not uniform, we cannot expect an exact coincidence of the timedependent tunneling currents with those corresponding to a uniform field configuration.

\subsection{Multiquantum well structure with two magnetic quantum wells}

We have seen that dipole waves are generated at magnetic QWs. Depending on the voltage, magnetizing a second QW, $i_{2}$ can inhibit nucleation of dipole waves at the first magnetic QW at $i_{1}, 1<i_{1}<i_{2}$. See the density plots in figure 12 , which have been calculated with the following 


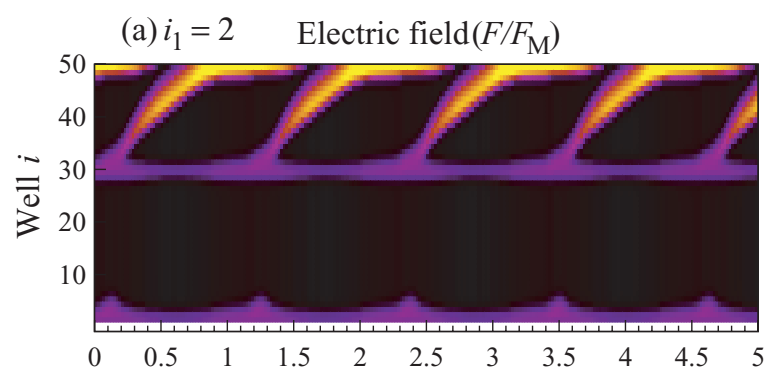

(c) $i_{1}=13$

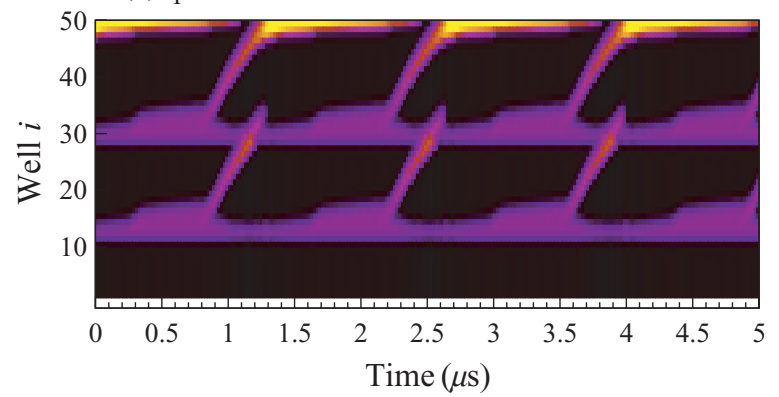

(b) $i_{1}=9 \quad$ Electric field $\left(F / F_{\mathrm{M}}\right)$

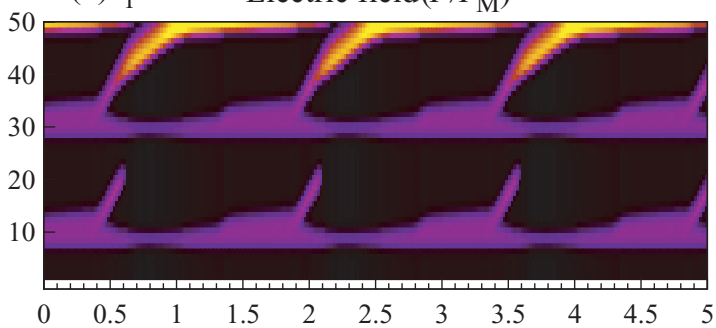

(d) $i_{1}=20$

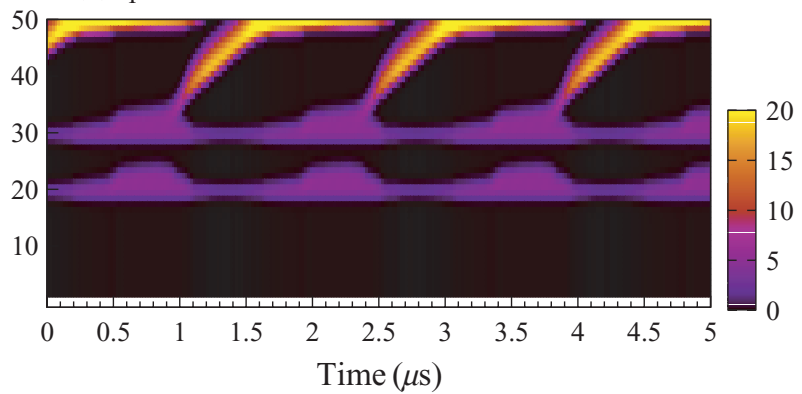

Figure 12. Density plot of the electric field profile during SSCOs for $i_{2}=30$, $N=50$ and (a) $i_{1}=2$, (b) $i_{1}=9$, (c) $i_{1}=13$ and (d) $i_{1}=20$. In (a) and (d), dipole waves can only move between QWs, $i_{2}=30$ and $N=50$. In (b) and (c) dipole waves are also nucleated at $i_{1}$. Other parameter values are $N_{\mathrm{D}}=$ $10^{10} \mathrm{~cm}^{-2}, \Delta=15 \mathrm{meV}$ and $\phi=2$. The apparent pixelation in this figure reflects the fact that the QW index is discrete.

parameter values: $N=50, i_{2}=30, N_{\mathrm{D}}=10^{10} \mathrm{~cm}^{-2}, \Delta=15 \mathrm{meV}, \phi=2$ (first voltage interval of SSCOs). For $i_{1}$ too close to either $i=1$ or to $i_{2}$, waves can move only from $i_{2}$ to $N$; see figures 12(a) and (d). For intermediate values of $i_{1}$, dipole waves are also nucleated at $i_{1}$, they move towards $i_{2}$ and either vanish before reaching $i_{2}$ (as in figure 12(b)) or, for $i_{1}$ above the critical value 13 (which is about half $i_{2}=30$ ), they reach and even surpass $i_{2}$ (as in figure 12(c)). For $i_{1}>13$ and sufficiently large, the dipole waves triggered at $i_{1}$ again vanish before reaching $i_{2}=30$ and can even be inhibited as shown in figure 12(d). Inhibition of dipole nucleation at one of the two magnetic QW disappears if the voltage is sufficiently large. For example, we have checked that waves are generated and move to the end of the MQWS if $\phi=2.7$ and $i_{1}=13$, instead of the inhibition effect shown in figure 12(b). We have also observed that for $i_{2}=30$, there is no inhibition of dipole nucleation in the special case $i_{1}=1$.

\subsection{Short multiquantum well structure}

We have investigated which is the shortest SL displaying self-oscillations in a configuration such that only the first QW is magnetic. For our parameter values, we find SSCOs for SL having 4 or more periods. Bonilla et al [15] show the total current density (most of which is due to spin-up electrons), the field and the spin polarization defined by (13) at the QWs during SSCOs for $N=4$. The first QW is always fully polarized, whereas the others are strongly polarized only when the dipole wave is traversing them: their spin polarizations drop abruptly afterward. The fraction of the oscillation period during which the $i$ th QW is strongly polarized decreases as $i$ increases. 


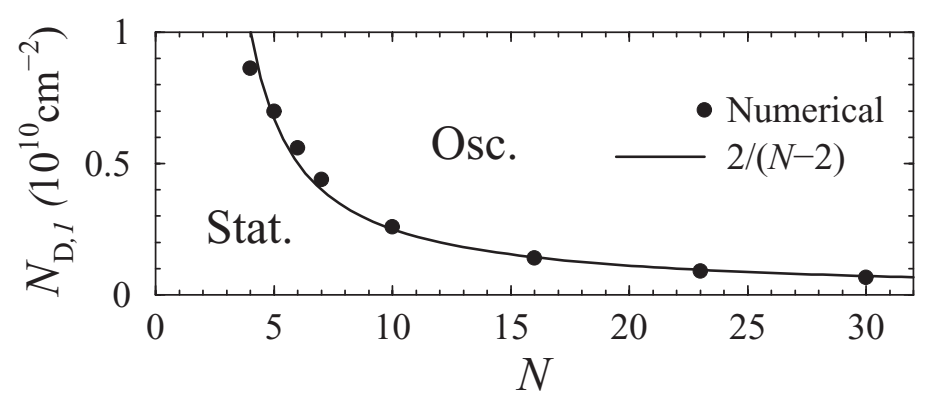

Figure 13. Minimal doping density for self-oscillations versus $N$.

For fixed $N \geqslant 4$, SSCOs appear if $N_{\mathrm{D}}>N_{\mathrm{D}, 1}$, where this first critical doping density is $N_{\mathrm{D}, 1}=[2 /(N-2)] \times 10^{10} \mathrm{~cm}^{-2}[15]$. We have checked this formula for $N \leqslant 50$; see figure 13 . In the continuum limit as $N \rightarrow \infty$, this yields $N N_{\mathrm{D}, 1} \approx 2 \times 10^{10} \mathrm{~cm}^{-2}$ which corresponds to the N-L criterion in the theory of the Gunn effect [22]. Different from bulk devices, our MQW structure is spatially discrete and moving accumulation layers (which are charge monopoles, forming part of moving dipoles) can be pinned by the lattice thereby yielding static EFDs [25]. Then there is a second critical doping density above which SSCOs disappear. Following [25], we find a bound which is the solution $N_{\mathrm{D}}=N_{\mathrm{D}, 2}$ of the following equation:

$$
\begin{aligned}
& N_{\mathrm{D}}+2 \rho \ln \left[1+\mathrm{e}^{-\left(e F_{\min } l / k_{\mathrm{B}} T\right)}(\Phi-1)\right]=\frac{J_{\mathrm{M}} l}{e v^{(f)}\left(F_{\min }\right)}-\frac{\varepsilon}{e}\left(F_{\min }-F_{\mathrm{M}}\right), \\
& \ln \Phi=\frac{2}{\rho}\left[N_{\mathrm{D}}+\frac{\varepsilon}{e}\left(F^{(3)}\left(J_{\mathrm{M}}\right)-F_{\min }\right)\right] .
\end{aligned}
$$

Here, $F_{\min }>0$ (independent of $N_{\mathrm{D}}$ ) is the field at which $F v^{(f)}(F)$ reaches its first relative minimum after its local maximum, and $J_{\mathrm{M}}$ and $F_{\mathrm{M}}$ are the coordinates of the maximum of the tunneling current at a nonmagnetic QW provided $n_{i}^{ \pm}=n_{i+1}^{ \pm}=N_{\mathrm{D}} / 2$. For these values of the electron densities and for positive $F, \mathcal{J}(F)$ has two local maxima, of which only the first one is shown in figure 9; the second maximum can be seen in figure 1(d) of [15]. Thus for appropriate values of $J$, the equation $\mathcal{J}(F)=J$ has four positive solutions $F^{(j)}(J)$ with $F^{(1)}<F^{(2)}<F^{(3)}<F^{(4)}$. $F^{(3)}(J)$ is the third one of these solutions.

For our parameter values, we obtain

$$
N_{\mathrm{D}, 2}=7.91 \times 10^{11} \mathrm{~cm}^{-2},
$$

which agrees reasonably well with numerical solutions of (1)-(9). If $10 \leqslant N \leqslant 50$, there exist stationary field profiles with two EFDs separated by a charge accumulation layer for $N_{\mathrm{D}} \geqslant 10^{11} \mathrm{~cm}^{-2}$. Beyond a doping density $\approx 7.96 \times 10^{11} \mathrm{~cm}^{-2}$, a field profile with a high-field domain separated from the contacts by low-field domains appears and SSCOs do not occur.

\section{Conclusions}

We have studied the phase diagram, current-voltage characteristics and SSCOs for a MQWS with one or two QWs doped with magnetic impurities. If only the first QW contains magnetic impurities, there are voltage intervals where there are stable SSCOs separated by intervals of 
stable stationary states for appropriate doping density. The first voltage interval of SSCOs is the widest and SSCOs are due to periodic triggering of dipole waves at magnetized QWs. In this interval, the motion of dipoles is confined to part of the structure for lower voltages and it arrives at the structure end for intermediate voltages. For larger voltages, there is a charge accumulation at the end of the MQWS and motion of dipoles is confined between the magnetized well and the charge accumulation layer. The branch of SSCOs ends by its collision with a homoclinic orbit of the stationary state. To understand the SSCOs, we have to realize that the magnetized well acts as a boundary condition creating a charge depletion layer in its neighborhood. When the tunneling current density from this well reaches the same value as the total current density, a dipole wave is nucleated there and released afterwards when these two currents again coincide. If two wells are magnetized in a long MQWS, each tries to trigger dipole waves, but, depending on the voltage, creation of waves at the QW farther from the cathode may inhibit dipole nucleation at the other one.

During SSCOs, QWs are fully polarized when the dipole wave is traversing them. Therefore, a short device (with four wells) would periodically inject pulses of polarized current to the collector. It is important that normal contacts can be used to build the oscillator, because the crucial requirement is to dope the first QW with $\mathrm{Mn}$. We have also indicated the range of doping density needed to achieve spin-polarized SSCOs. For self-oscillations to occur, appropriate ranges of spin splitting should be induced by tailoring the magnetic impurity density and external magnetic fields. Our results could be used to construct an oscillatory spin-polarized current injector.

\section{Acknowledgments}

This work has been supported by the MECD grants MAT2005-05730-C02-01 and MAT200506444. R E thanks the Ramón y Cajal Program of the Spanish Ministry of Education and Science.

\section{References}

[1] Bandyopadhyay S and Cahay M 2008 Introduction to Spintronics 2nd edn (Boca Raton, FL: CRC Press)

[2] Wolf S A, Awschalom D D, Buhrman R A, Daughton J M, von Molnár S, Roukes M L, Chtchelkanova A Y and Treger D M 2001 Science 2941488

[3] Awschalom D D and Flatté M E 2007 Nat. Phys. 3153

[4] Schmidt G, Ferrand D, Molenkamp L W, Filip A T and van Wees B J 2000 Phys. Rev. B 62 R4790

[5] Zutic I, Fabian J and Erwin S C 2007 J. Phys.: Condens. Matter 19165219 Zutic I, Fabian J and Das Sarma S 2004 Rev. Mod. Phys. 76323

[6] Fabian J, Matos-Abiague A, Ertler C, Stano P and Zutic I 2007 Acta Phys. Eslovaca 57565

[7] Egues J C 1998 Phys. Rev. Lett. 804578

[8] Fiederling R, Kleim M, Reuscher G, Ossau W, Schmidt G, Waag A and Molenkamp L W 1999 Nature 402787

Ono Y, Young D K, Beschoten B, Matsukura F, Ohno H and Awschalom D D 1999 Nature 402790

[9] Khaetskii A, Egues J C, Loss D, Gould C and Schmidt G 2005 Molenkamp L W Phys. Rev. B 71235327

Smith D L and Silver R N 2001 Phys. Rev. B 64045323

Schmidt G, Gould C, Grabs P, Lunde A M, Richter G, Slobodskyy A and Molenkamp L W 2004 Phys. Rev. Lett. 92226602 
[10] Slobodskyy A, Gould C, Slobodskyy T, Schmidt G, Molenkamp L W and Sánchez D 2007 Appl. Phys. Lett. 90122109

[11] Mireles F and Kirczenow G 2002 Phys. Rev. B 66214415

[12] Crooker S A, Tulchinsky D A, Levy J, Awschalom D D, Garcia R and Samarth N 1995 Phys. Rev. Lett. 75505

Berry J J, Knobel R, Ray O, Peoples W and Samarth N 2000 J. Vac. Sci. Technol. B 181692

[13] Sánchez D, MacDonald A H and Platero G 2002 Phys. Rev. B 65035301

[14] Béjar M, Sánchez D, Platero G and MacDonald A H 2003 Phys. Rev. B 67045324

[15] Bonilla L L, Escobedo R, Carretero M and Platero G 2007 Appl. Phys. Lett. 91092102

[16] Slobodskyy A, Gould C, Slobodskyy T, Becker C R, Schmidt G and Molenkamp L W 2003 Phys. Rev. Lett. 90246601

[17] Bonilla L L 2002 J. Phys.: Condens Matter 14341

[18] Xu H and Teitsworth S W 2007 Phys. Rev. B 76235302

[19] Kikkawa J M, Smorchkova I P, Samarth N and Awschalom D D 1997 Science 2771284

[20] Awschalom D D and Samarth N 1999 J. Magn. Magn. Mater. 200130

[21] Bonilla L L, Escobedo R and Higuera F 2001 Phys. Rev. E 65016607

[22] Kroemer H 1972 Topics in Solid State and Quantum Electronics ed W D Hershberger (New York: Wiley) p 20

[23] Bonilla L L and Grahn H T 2005 Rep. Prog. Phys. 68577

[24] Bonilla L L, Platero G and Sánchez D 2000 Phys. Rev. B 622786

Sánchez D, Moscoso M, Bonilla L L, Platero G and Aguado R 1999 Phys. Rev. B 604489

[25] Carpio A, Bonilla L L, Wacker A and Schöll E 2000 Phys. Rev. E 614866 